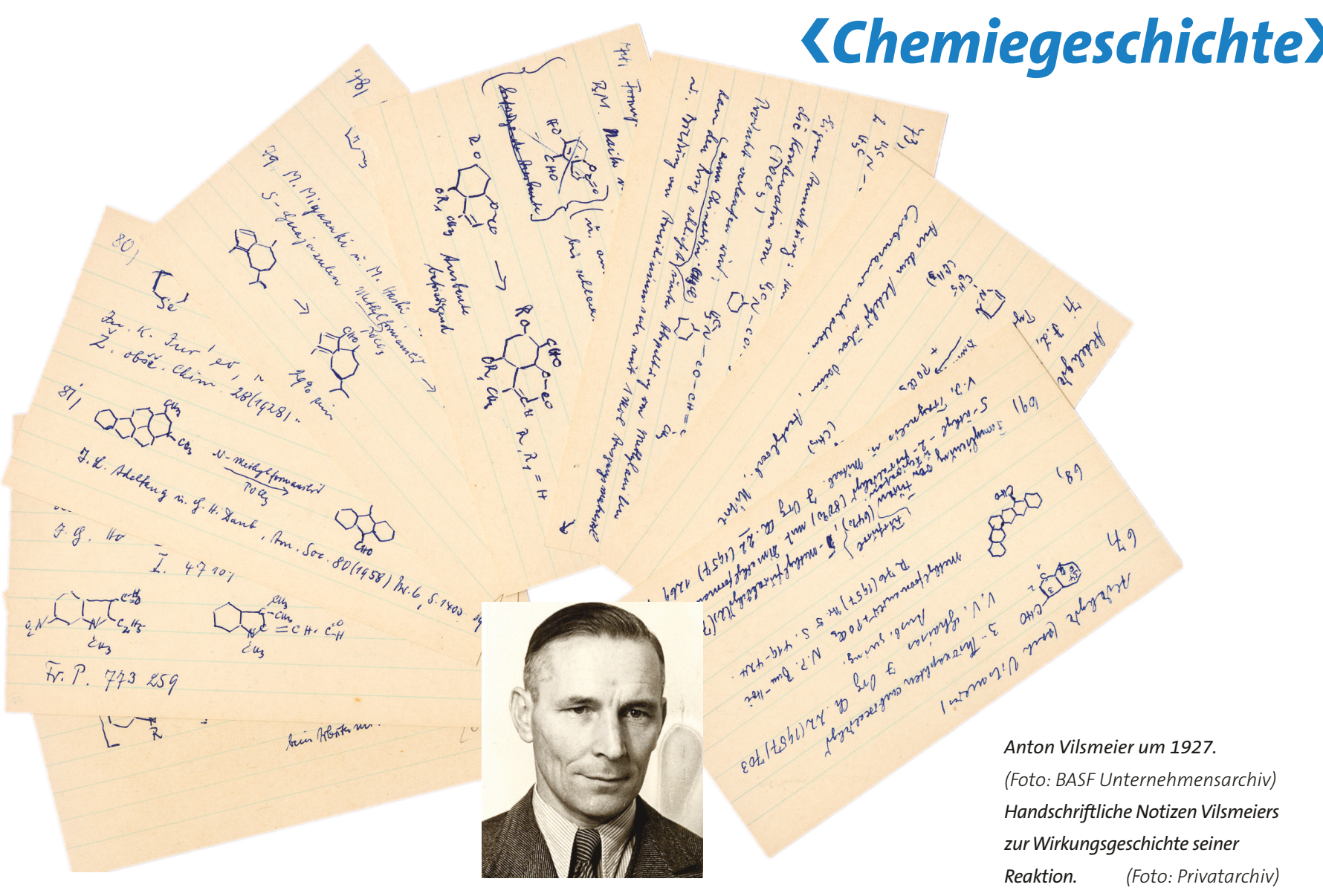

\title{
Ein Mann und seine Reaktion
}

\section{Christoph Meinel}

Industriechemiker machen dem Biographen die Arbeit nicht leicht. Sie publizieren in Patenten und internen

Berichten und nicht in Fachzeitschriften. Sie haben kaum Teil an den Ritualen akademischer Erinnerungs-

kultur. Deshalb ist auch wenig über den Mann bekannt, der hinter der Reaktion steht, der er seinen Namen

gab: Anton Vilsmeier (1894-1962).

- Namensreaktionen sind Reaktionen, „die man aus historischen Gründen, wegen ihrer fortdauernden Bewährung in der Praxis und... wegen der damit verknüpften Vorteile mit dem Namen ihrer Entdecker bezeichnet". 1) Gegenüber den praktischen Vorzügen der Kurzformel tritt das historische Interesse so weit zurück, dass der Name zur bloßen Chiffre wird und die Person hinter dem Namen verschwindet. Dies trifft auch für den Namensgeber der Vilsmeierschen Aldehydsynthese zu, über den sich - mit
Ausnahme einer Notiz in der Erlanger Universitätszeitung von 19992) - in der Literatur keine Angaben finden.

Anton Vilsmeier wurde am 12. Juni 1894 in Burgweinting geboren, seit 1977 ein Stadtteil von Regensburg. Der Vater Wolfgang Vilsmeier war Landwirt und Mühlenbesitzer, Anton das zweite von zwölf Kindern. ${ }^{3)}$ Vier von ihnen haben später studiert, drei sogar promoviert. In Burgweinting besuchte Anton die Volksschule, von 1905 an das Gymnasium. Im Jahr 1912 brach der 17-jährige Anton mit der römisch-katholischen Kirche und verließ am 30. April aus eigenen Stücken sowohl das Studienseminar St. Emmeram als auch das Gymnasium. Es folgte eine Lehre bei der Bayerischen Vereinsbank in Passau, die er nach anderthalb Jahren abbrach, um sich autodidaktisch auf das Abitur vorzubereiten. Dazu konnte er, der bayerischen Schulordnung zufolge, als Privatstudierender ohne weiteren Schulbesuch zugelassen werden. So unterzog er sich 1914 an seinem früheren Gymnasium dem Absolutorium. 43 Abiturien- 
ten traten zur Prüfung an: Vierzehn davon gaben als Berufsziel katholische Theologie an, vier wollten evangelische Pfarrer werden, vier Jura und sechs Medizin studieren, vier den „militärischen Beruf" ergreifen und drei sich der Forstwissenschaft zuwenden - zu letzteren gehörte auch Anton Vilsmeier.

Bei fast allen dürfte der Krieg diese Pläne vereitelt oder zumindest verzögert haben. Vilsmeier wurde zum 11. Bayerischen Infanterie-Regiment und im Januar 1915 an die Front einberufen. Während der Somme-Schlacht geriet er im September 1916 in englische Gefangenschaft, aus der er im November 1919 entlassen wurde.

\section{Studium und Assistentenzeit}

- Im Februar 1920 schrieb Vilsmeier sich als Chemiestudent an der Münchner Universität ein; zum Wintersemester 1922/23 wechselte er an die Universität Erlangen. Dort leitete der Farbstoffchemiker Otto Fischer (18521932) das Laboratorium. Hier legte Vilsmeier das Erste und Zweite Verbandsexamen $a b^{4)}$ - eine frei-

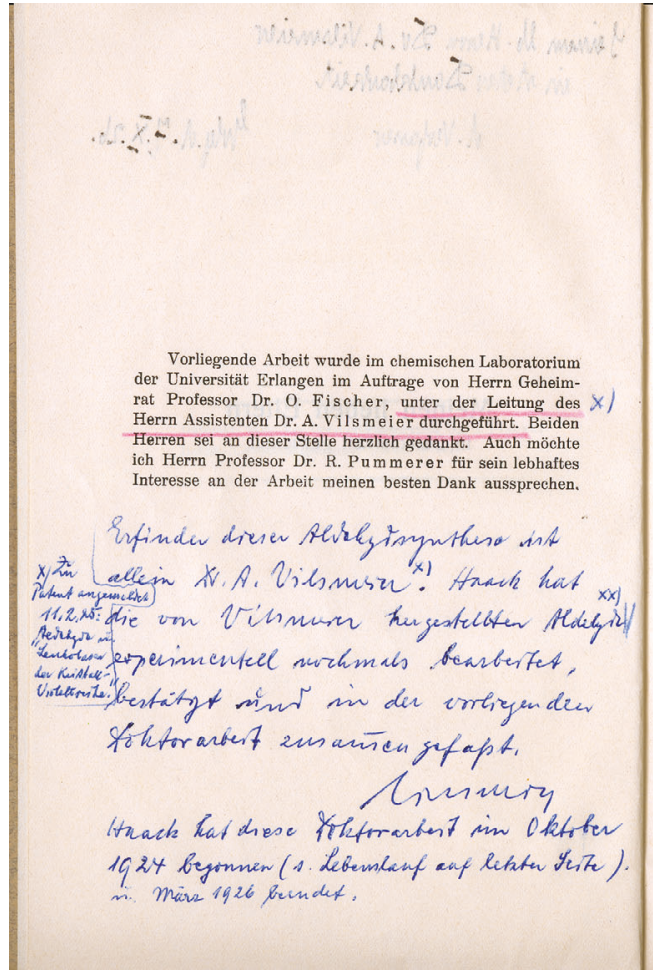

Albrecht Haacks Dissertation mit Widmung an Vilsmeier. ${ }^{8)}$

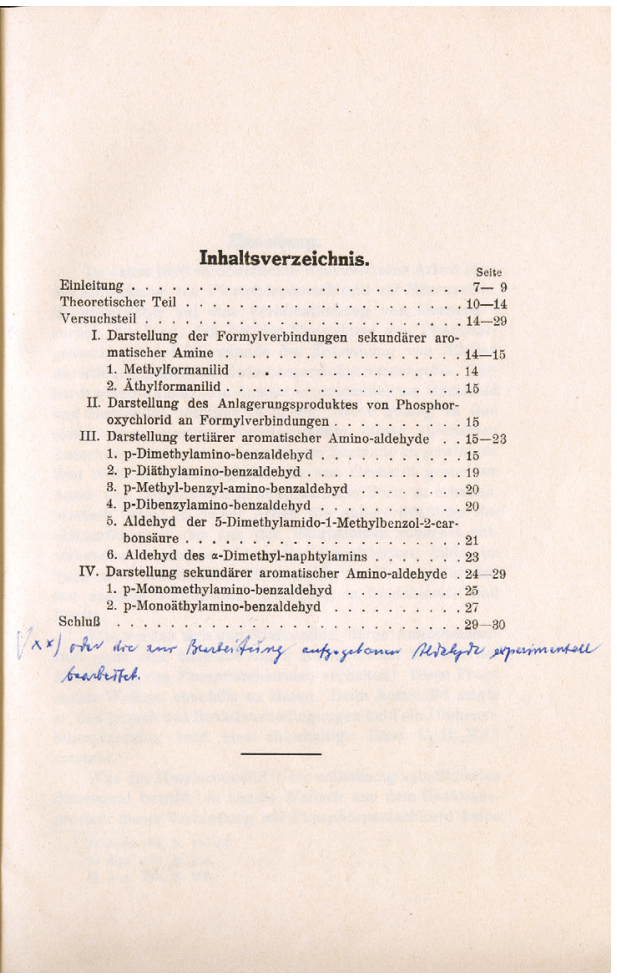

(Foto: Privatarchiv)

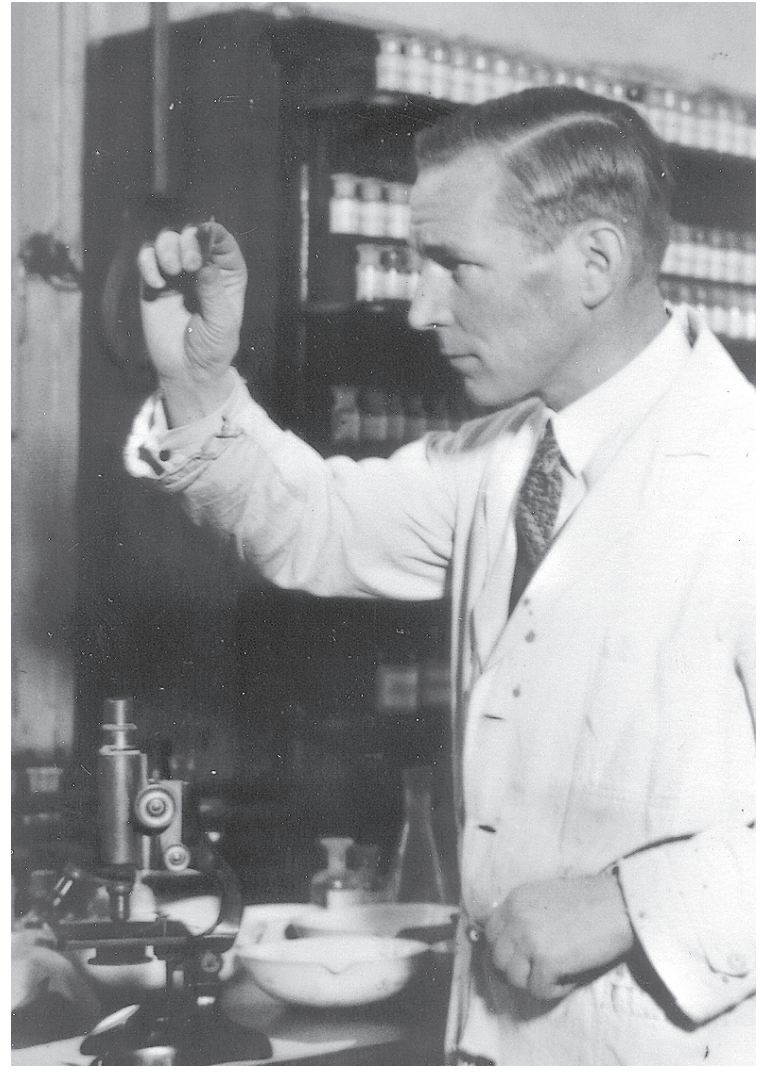

willige Prüfung, die dazu berechtigte, die Arbeit an der Dissertation aufzunehmen; denn eine universitäre Abschlussprüfung für Chemiker gab es damals noch nicht.

smeier als Assistent in Erlangen. (Foto: Privatarchiv)

Seit Dezember 1923 als Assistent bei Fischer beschäftigt, promovierte Vilsmeier am 30. Juli 1924 zum Dr. phil. In der 15-seitigen, maschinenschriftlich vervielfältigten Arbeit mit dem Titel „ $\gamma$-Chlor-IsoChinocyanine aus Methyl-(Aethyl-) Acetanilid und Phosphoroxychlorid" hatte Vilsmeier die Struktur eines 1894 von Charles Friedel (1832-1899) beschriebenen roten Farbstoffs untersucht und diesen als Chinolin-Derivat identifiziert. ${ }^{5)}$ Ende des Wintersemesters 1925 wechselte Vilsmeier als Assistent zu Fischers Nachfolger, dem Farbstoffchemiker Rudolf Pummerer (1882-1973). Mit Ausnahme der Ergebnisse seiner Dissertation ${ }^{6)}$ hat Vilsmeier weder mit Fischer noch mit Pummerer gemeinsam publiziert.

Offenbar nutzte Vilsmeier seine Assistentenzeit auch für die Aufklärung des in seiner Doktorarbeit untersuchten Reaktionsmechanismus. So ließ er vom Oktober 1924 an die von ihm gefundene Aldehydsynthese durch den Doktoranden Albert Haack (1898-1976) untersuchen. Ausgehend von N-Me- 
thyl- oder N-Ethyl-Formanilid wurde dabei als reaktive Zwischenstufe zunächst das Anlagerungsprodukt aus $\mathrm{POCl}_{3}$ und der Formylverbindung des sekundären Amins charakterisiert. Dieses Vilsmeier-Reagenz wurde dann zur Darstellung sekundärer und tertiärer aromatischer Amino-Aldehyde eingesetzt. Dabei zeigte sich, dass die Methode allgemein zur Formylierung elektronenreicher Aromaten geeignet ist. ${ }^{7)}$

Im Exemplar der gedruckten Dissertation, das Haack „Seinem lb. Herrn Dr. A. Vilsmeier in steter Dankbarkeit" zugeeignet hatte, ${ }^{8)}$ findet sich eine handschriftliche Notiz. In dieser stellt Vilsmeier klar, dass er der alleinige „Erfinder dieser Aldehydsynthese" sei, und die schon zuvor von ihm, Vilsmeier, hergestellten Aldehyde von Haack lediglich experimentell noch einmal bearbeitet, bestätigt und zusammengefasst worden seien. Zudem erklärt Vilsmeier, dass er die Aldehydsynthese am 11. Februar 1925 unter dem Betreff „Aldehyde und Leukobasen der Kristallviolettreihe" zum Reichspatent angemeldet habe. Die Erteilung eines entsprechenden Patents ist jedoch nicht nachweisbar.

\section{Farbstoffchemie bei der BASF}

- Der traditionelle Schwerpunkt des Erlanger Laboratoriums auf der Farbstoffchemie und nicht zuletzt die guten Kontakte seines Chefs Pummerer zur BASF dürften den Ausschlag gegeben haben, dass Anton Vilsmeier 1927 nach Ludwigshafen wechselte. Am 1. September trat er in das Hauptlabor der „Betriebsgemeinschaft Oberrhein" ein, wie der BASFStandort Ludwigshafen als Teil der I.G. Farbenindustrie damals hieß. ${ }^{9)}$

Die BASF war das führende Chemieunternehmen der Welt und eines der ersten, das systematisch eine eigene Forschungsabteilung aufgebaut hatte. ${ }^{10)}$ Als Vilsmeier in das Hauptlabor eintrat, arbeiteten dort etwa 50 promovierte Chemi- ker. Nach Jahresfrist wechselte er in die Alizarin-Abteilung und zwei Jahre später in deren wissenschaftliches Labor: mit mehr als 20 Chemikern das zweitgrößte Forschungs- und Entwicklungslabor des Unternehmens. ${ }^{11)}$

Im Zentrum standen hier die lichtechten Küpenfarbstoffe aus der Indanthren-Reihe. Aus diesem Arbeitsgebiet sollten bis 1940 mehr Patente hervorgehen als aus jedem anderen Labor der I.G. ${ }^{12)}$ Vilsmeier selbst arbeitete an der technischen Herstellung von Indanthrenrot FBB. In den 1930er Jahren folgten Erstsynthesen und Verfahrensentwicklung weiterer Küpenfarbstoffe. Hinzu kamen Arbeiten über Metallkomplexfarbstoffe, deren Ergebnisse in der Azound Textilabteilung des Unternehmens weiterentwickelt wurden. Niedergelegt hat Vilsmeier die Ergebnisse in Patentschriften - zwischen 1930 und 1939 firmiert er auf 19 Farbstoffpatenten - oder in werksinternen Berichten und Fabrikationsvorschriften. ${ }^{13)}$ Während der übliche Karriereweg eines Industriechemikers nach wenigen Jahren der Forschung in die Produktion oder ins Management führte, blieb Vilsmeier die gesamte Zeit seiner Berufstätigkeit hindurch Forschung und Entwicklung verbunden.

Die Veränderungen der deutschen Chemieindustrie in jener Zeit scheinen das Alizarin-Labor wenig tangiert zu haben. Wie sich die Weltwirtschaftskrise auf die tägliche Arbeit ausgewirkt hat, und wie später das Primat der Rüstungsproduktion in Vilsmeiers unmittelbarem Arbeitsumfeld zu spüren war, ist schwer zu sagen. In die Kriegswirtschaft lassen sich seine eigenen Arbeiten jedenfalls nicht einordnen. Und darüber, wie Vilsmeier selbst zu der Politisierung der Belegschaft und den Verbindungen der Werksleitung zur politischen Führung stand, liegen keine Quellen vor - für eine Mitgliedschaft Vilsmeiers in NS-Organisationen finden sich keine Hinweise. ${ }^{14)}$

\section{Kriegs- und Nachkriegszeit}

- Offensichtlich war das Wissenschaftliche Labor der Alizarin-Abteilung nicht kriegswichtig genug, und so wurde Vilsmeier von Juni 1941 bis November 1944 zur Wehrmacht eingezogen. Aus den Unterlagen der BASF geht hervor, dass Vilsmeier im November 1944 zurückgeholt und in das I.G.-Werk Dyhernfurth nördlich von Breslau beordert wurde. Er hatte dort die Aufgabe, Produktionsanlagen abzubauen, um sie dem Zugriff der vorrückenden alliierten Armeen zu entziehen. Der gleichen Aufgabe kam er von Februar bis April 1945 im I.G.-Werk Gendorf im Kreis Altötting nach. Beide Betriebe hatten während des Krieges der Produktion chemischer Kampfstoffe gedient. ${ }^{15)}$

In Ludwigshafen erreichten die amerikanischen Panzer am 23. März 1945 das Werksgelände. Nach den Bombenangriffen im zweiten Halbjahr 1944 war die Produktion auf zehn Prozent des ursprünglichen Niveaus zurückgegangen, im Februar 1945 kam sie praktisch zum Stillstand, und von den Anlagen war nur noch ein Drittel intakt. ${ }^{16)}$ Vilsmeier, vom 1. Mai bis zum 26. Juni in amerikanischer Gefangenschaft, kehrte am 28. August 1945 nach Ludwigshafen zurück und wurde wieder dem Alizarin-Labor zugeordnet. Industriepolitisch begann nun die Zeit der Entflechtung des I.G. FarbenKonzerns, aus dem im Januar 1952 die BASF wieder als eigenständiges Unternehmen hervorging. ${ }^{17)}$ Zum gleichen Zeitpunkt, am 1. Januar 1952, wechselte Vilsmeier in deren Farbenlabor.

Seine chemischen Arbeiten setzten dort ein, wo sie der Krieg unterbrochen hatte. Im Zentrum standen wieder die Küpenfarbstoffe aus der Anthrachinon-Reihe; außerdem ging es um Zwischenprodukte für ihre Synthese und um Verfahren zum Weißätzen, also zur Nachbehandlung von bereits auf die Textilfaser aufgezogenen Küpenfarbstoffen. Am 1. Oktober 1948 
erhielt Vilsmeier das erste Nachkriegspatent: auf Indanthrenrotbraun $G$ und Indanthrenmarron BR. ${ }^{18)}$ Bis 1959 folgten 15 weitere Patentanmeldungen. ${ }^{14)}$

Am 1. Juli 1959 trat Anton Vilsmeier in den Ruhestand, vor 50 Jahren, am 12. Februar 1962, ist er in Ludwigshafen gestorben.

\section{Vilsmeier und „seine“ Reaktion}

- Wer war der Mensch hinter der Vilsmeier-Reaktion? Zu einem wirklichen Persönlichkeitsbild fügen sich die Mosaiksteinchen seines Lebensweges nicht. Wie Vilsmeier das Ende der Weimarer Republik, den Aufstieg des Nationalsozialismus, wie er Krieg und Nachkriegszeit und das Wirtschaftswunder der 1950er Jahre erlebt hat - wir wissen es nicht.

Bis mindestens 1930 war Vilsmeier Mitglied der Deutschen Chemischen Gesellschaft und außerdem Mitglied des Vereins Deutscher Chemiker, des Berufsverbands der Industriechemiker. Doch eine besondere Funktion hat er in diesen Vereinigungen nicht ausgeübt. Anton Vilsmeiers Sohn Wolfgang berichtet, sein Vater sei ein überzeugter Humanist und Kenner der griechisch-römischen Antike gewesen, sei zur Jagd gegangen und habe die Musik geliebt. Selbstzeugnisse fehlen.

Die Formylierungsreaktion, die am Anfang seines wissenschaftlichen Weges gestanden hatte, hat Vilsmeier weiterhin im Auge behalten. In einem Übersichtsartikel, der 1951 in der Chemiker-Zeitung erschien ${ }^{19)}$ - seine einzige Zeitschriftenpublikation seit den beiden Aufsätzen aus der Erlanger Zeit - berichtet er nicht ohne Stolz über die Anwendungen, die der Reaktionstyp sowohl in der Forschung wie auch industriell gefunden hatte. Und in seinem Nachlass fand die Familie Karteikarten, auf denen Vilsmeier bis 1960 die Schicksale der von ihm entdeckten Reaktion verfolgt hat.

Der Wissenschaftshistoriker und Chemiker Christoph Meinel, Jahrgang 1949, ist Inhaber des Lehrstuhls für Wissenschaftsgeschichte an der Universität Regensburg. Anlässlich der ersten Anton-Vilsmeier-Vorlesung der Fakultät für Chemie und Pharmazie der Universität Regensburg im Juni 2012 begab er sich auf Spurensuche zu dem Mann hinter der Reaktion.christoph.meinel@psk.uni-regensburg.de

Literatur

1) F. Richter, „Geleitwort [1960],“in: $\mathrm{H}$. Krauch, W. Kunz, Reaktionen der organischen Chemie, 6. Aufl. [Hrsg.: W. Kunz, E. Nonnenmacher], Heidelberg 1997.

2) W. R. Pötsch, „FAU-Assistent Anton Vilsmeier fand Aldehydsynthese, "FAU UniKurier Aktuell 6/27 (Juli 1999), 8.

3) Wichtigste Quellen zur Biographie Vilsmeiers sind der Lebenslauf in seiner maschinenschriftlichen Dissertation ${ }^{5)}$ sowie persönliche Mitteilungen seines Sohnes Wolfgang Vilsmeier, Ditzingen, mit Unterlagen aus dem Besitz der Familie.

4) Das vom "Verband der Laboratoriumsvorstände an deutschen Hochschulen" 1897 eingeführte Examen wurde üblicherweise in zwei Teilen abgelegt, wobei der organische den Abschluss bildete. Siehe: J. A. Johnson, „Academic Self-Regulation and the Chemical Profession in Imperial Germany," Minerva, 1985, 23, 241-271.

5) A. Vilsmeier, „r-Chlor-Iso-Chinocyanine aus Methyl-(Aethyl-)-Acetanilid und Phosphoroxychlorid," Phil. Diss., Universität Erlangen, 1924 [masch.schr.].

6) O. Fischer, A. Müller, A. Vilsmeier, „Über die Einwirkung von Phosphoroxychlorid auf Methyl-(Äthyl-)-acetanilid: Synthesen von $\gamma$-Chlor-iso-chinocyaninen," Journal für praktische Chemie, 1925 , 109, 69-87.
7) A. Vilsmeier, A. Haack, „Über die Einwirkung von Halogenphosphor auf AlkylFormanilide: Eine neue Methode zur Darstellung sekundärer und tertiärer $p$-Alkyl-amino-benzaldehyde, "Berichte der Deutschen Chemischen Gesellschaft 1927, 60, 119-122.

8) A. Haack, „Über die Einwirkung von Phos phorchloriden $\left(\mathrm{POCl}_{3}, \mathrm{PCl}_{5}\right)$ auf Methyl(Äthyl-) Formanilid: Synthese sekundärer und tertiärer aromatischer Amino-aldehyde, "Phil. Diss. Universität Erlangen 1926. [Exemplar im Besitz der Familie.]

9) Mit gleichem Eintrittsdatum wurde Vilsmeier unter der Nummer 545 auf der Chemiker-Liste der „Betriebsgemeinschaft Oberrhein" geführt. Persönliche Mitteilung von Jeffrey A. Johnson, Villanova/PA, vom 15.2.2012.

10) C. Reinhardt, Forschung in der chemischen Industrie: Die Entwicklung synthetischer Farbstoffe bei BASF und Hoechst, 1863-1914, Freiberg 1997; C. Reinhardt, A. S. Travis, Heinrich Caro and the Creation of Modern Chemical Industry, Dordrecht 2000.

11) Angaben und Daten aus der Personalakte, persönliche Mitteilung von Susan Becker, BASF Unternehmensarchiv, vom 22.2.2012.

12) Erfindertätigkeit in der BASF: Statistiken von 1877-1940, BASF Unternehmensarchiv, E05/1.

13) A. Vilsmeier, „Lebenslauf“ / "Aufstellung meiner chemischen Arbeiten und Veröffentlichungen, 5. November 1945, “5 Bll., masch.schr., Unternehmensarchiv der BASF. Ferner: [A. Vilsmeier,] „Patentanmeldungen, an denen Herr Dr. A. Vilsmeier erfinderisch beteiligt ist, 9. Januar 1952" (mit Nachträgen vom 14. Februar 1962), 4 Bll., masch.schr., Unternehmensarchiv der BASF.

14) Mitteilung von Thomas Steinhauser, Berlin, sowie dem Bundesarchiv vom 22.2.2012.

15) F. Schmaltz, Kampfstoff-Forschung im Nationalsozialismus: Zur Kooperation von Kaiser-Wilhelm-Instituten, Militär und Industrie, Göttingen 2005, S. 159-162, 449-456.

16) R. G. Stokes, „Von der I.G. Farbenindustrie AG bis zur Neugründung der BASF, 1925-1952,“ in: Die BASF: Eine Unternehmensgeschichte [Hrsg.: W. Abelshauser], 2. Aufl., München 2003, S. 221-358.

17) W. Abelshauser, „Die BASF seit der Neugründung 1952,“ in: Die BASF [wie ${ }^{16)}$ ], S. 359-634.

18) K. Saftien, A. Vilsmeier, „Verfahren zur Herstellung von Küpenfarbstoffen der Pyranthronreihe, "DRP 15334 (1.10.1948).

19 A. Vilsmeier, „Über die Herstellung von Aldehyden mit Hilfe von N-disubstituiertem Formamid," Chemiker-Zeitung 1951, 75, 133-135. 